\title{
High Intensity Positron Program at LLNL
}

\author{
P. Asoka-Kumar \\ R. Howell \\ W. Stoeffl
}

This paper was prepared for submittal to the

\section{Conference on Applications of Accelerators in Research and Industry}

Denton, $T X$

Nevember 4-7, 1998

September 23, 1998

This is a preprint of a paper intended for publication in a journal or proceedings. Since changes may be made before publication, this preprint is made available with the understanding that it will not be cited or reproduced without the permission of the author. 


\section{DISCLAIMER}

This document was prepared as an account of work sponsored by an agency of the United States Government. Neither the United States Government nor the University of California nor any of their employees, makes any warranty, express or implied, or assumes any legal liability or responsibility for the accuracy, completeness, or usefulness of any information, apparatus, product, or process

disclosed, or represents that its use would not infringe privately owned rights. Reference herein to any specific commercial product, process, or service by trade name, trademark, manufacturer, or otherwise, does not necessarily constitute or imply its endorsement, recommendation, or favoring by the United States Government or the University of California. The views and opinions of authors expressed herein do not necessarily state or reflect those of the United States Government or the University of California, and shall not be used for advertising or product endorsement purposes. 


\title{
High Intensity Positron Program at LLNL
}

\author{
P. Asoka-Kumar, R. Howell, W. Stoeffl, and D. Carter \\ Lawrence Livermore National laboratory, Livermore, CA 94550
}

\begin{abstract}
Lawrence Livermore National Laboratory (LLNL) is the home of the world=s highest current beam of $\mathrm{keV}$ positrons. The potential for establishing a national center for materials analysis using positron annihilation techniques around this capability is being actively pursued. The high LLNL beam current will enable investigations in several new areas. We are developing a positron microprobe that will produce a pulsed, focused positron beam for 3-dimensional scans of defect size and concentration with submicron resolution. Below we summarize the important design features of this microprobe. Several experimental end stations will be available that can utilize the high current beam with a time distribution determined by the electron linac pulse structure, quasi-continuous, or bunched at $20 \mathrm{MHz}$, and can operate in an electrostatic or (and) magnetostatic environment. Some of the planned early experiments are: two-dimensional angular correlation of annihilation radiation of thin films and buried interfaces, positron diffraction holography, positron induced desorption, and positron induced Auger spectroscopy.
\end{abstract}

\section{INTRODUCTION}

Positron annihilation spectroscopy has emerged as a highly sensitive, nondestructive probe to study the nature, concentration, and spatial distribution of defects in materials. ${ }^{1}$ Since the early 1970's, considerable efforts went into developing low-energy, monochromatic positron beams that can perform depthresolved defect analysis of materials. The underlying physical process for converting the positrons emitted from either a radio isotope or a stopping target in an electron linac into a monochromatic beam, known as Amoderation,@ is not very efficient (with a yield of about one positron for every $10^{3}$ positrons entering the moderator material). Therefore, most laboratory beams lack the brightness necessary to realize the full potential of this probe. The present status in our field is analogous to the one experienced by researchers using laboratory X-ray sources before the advent of synchrotron light sources. In this paper, we describe the efforts now underway at LLNL to remedy this situation by building a multiuser experimental program around an existing high current source of moderated positrons. ${ }^{2}$

\section{HIGH INTENSITY POSITRON BEAM}

The LLNL primary source of positrons is built at the end of a $100 \mathrm{MeV}$ linac, capable of producing $1 \mathrm{~A}$ of electrons at a repetition rate of $330 \mathrm{~Hz}$. The energetic electrons are stopped in a water-cooled tungsten target providing a shower of bremstrahlung photons. The pair conversion of the Bremstrahlung photons yields an intense source of positrons. These positrons shine onto a set of well-annealed tungsten foils (25 microns thick) arranged in the form of AVenetian blinds.@ A fraction of the high energy positrons is slowed down in the tungsten foils and is reemitted as moderated positrons. These positrons are harvested and guided to an experimental hall with a
30 gauss axial magnetic field. The beam transport system contains a curved section to prevent direct line of sight from the source region. The experimental hall and the positron production target are separated by $4.5 \mathrm{~m}$ of shielding to ensure a low radiation background environment in the experimental hall. Figure 1 shows the layout of the experimental hall.

The positron beam entering the experimental hall consists of short bursts of high intensity positrons with an energy width of $\sim 4 \mathrm{eV}$. The pulse duration and repetition rates are determined by the pulse structure of the electron linac, with nominal values of 3 :s and $300 \mathrm{~Hz}$, respectively. The intensity of the moderated positrons is determined by the energy, current, and focus quality of the electron beam. With $100 \mathrm{MeV}$ electron beam, every $3 \mathrm{H} 10^{5}$ electrons produce a moderated positron, resulting in a $10^{10}$ positrons $/ \mathrm{s}$ beam at full power.

The time structure of the initial positron beam is not well suited for many experiments. Every pulse contains $3 \mathrm{H} 10^{7}$ positrons and will saturate most detection systems. Therefore, the initial beam is captured, stored, and released slowly from a penning trap to produce a time-stretched beam profile. The pulse stretcher consists of 3 elements: a gate electrode at the entrance, a middle storage section (called Afloor@), and a barrier electrode at the exit. The voltage on the gate and barrier electrodes is higher than the primary positron energy of $\sim 15 \mathrm{~V}$, except for a short interval when the gate is switched low to let in the pulse of positrons. The positron bunch is thus captured between the gate electrode and barrier electrode. After filling the trap with a positron pulse, the voltage on the 


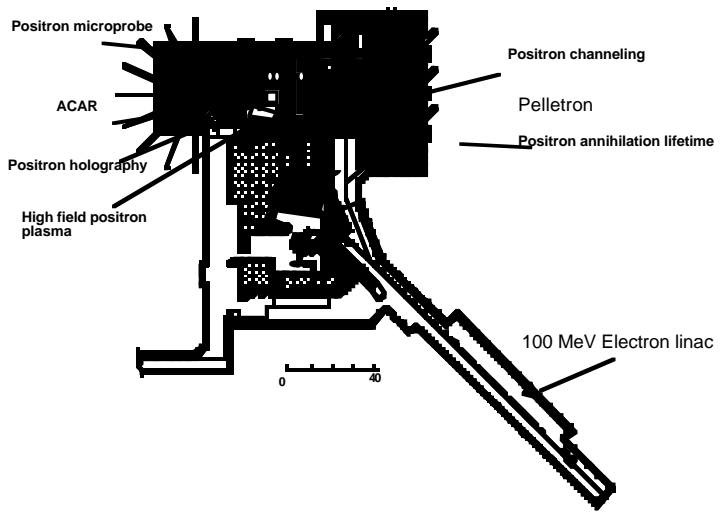

Figure 1. Layout of the intense positron beam at LLNL.

trap floor is raised slowly to spill the positrons over the fixed voltage barrier. The ramp voltage is adjusted to convert the initial 3 :s pulse into $\mathrm{a} \sim 3 \mathrm{~ms}$ pulse at the end of which the next bunch of positrons enters the trap to continue the sequence of events.

The trap efficiency was measured by capturing positrons and holding them for various times. The trap is then emptied by raising the floor and the positron yield is compared to the initial number of positrons. The primary positron intensity is obtained by turning off the barrier voltage. The positron yield is obtained by integrating the annihilation (-ray signal obtained with a NaI detector. The yield dropped to a $50 \%$ level when the positrons were held in the trap for $8 \mathrm{~ms}$, suggesting a positron trap life time of $11.5 \mathrm{~ms}$, at a base pressure of $3.0 \mathrm{H} 10^{-8}$ torr.

The energy spread of the beam spilling over the barrier is significantly smaller than the starting beam, $20 \mathrm{meV}$ versus $\sim 4 \mathrm{eV}$. The barrier is nominally set at 20 Volt and is followed by an accelerator column consisting of 30 electrodes, equally spaced in distance and voltage to provide a gentle acceleration of $20 \mathrm{eV}$.

Immediately following the accelerator is a two-gap time-buncher section that is pulsed to produce a nano second wide pulse every $50 \mathrm{~ns}$, and acts as the first pulsing stage of a positron microprobe (see later). Thus, depending on the user requirement, the stretcherbuncher configuration can deliver either a nearly continuous beam or a pulsed beam with well-defined time structure (containing $\sim 500$ positrons per pulse at $20 \mathrm{MHz}$ ). The stretcher and buncher can also be turned off to keep the time structure of the original linac beam.

\section{EXTRACTION OF POSITRONS FROM MAGNETIC FIELD}

Many experimental stations will require an electrostatic environment for optimum performance.
Therefore, the positrons have to be extracted out of the guiding magnetic field without significant degradation of the phase space. This is achieved with a magnetic grid consisting of 36 tapered fins pointing toward the center similar to the Aspokes@ of a wheel. As the spokes approach the center they are terminated at three different distances from the center to have an effective magnetic field termination and $>90 \%$ transmission. The magnetic fields are concentrated in the spokes and are guided outward by an iron shield. Tests show that at a distance of $2 \mathrm{~mm}$ from the magnetic grid, an internal magnetic field of 30 gauss is reduced to 0.3 gauss.

The motion of positrons crossing the magnetic field through the open space will be nonadiabatic. The positrons are accelerated to $2000 \mathrm{eV}$ with an accelerator section to increase the pitch length compared to the region in which the field lines are terminated. Figure 2 shows the positron beam emanating from the grid imaged onto a microchannel plate, located at a distance of $40 \mathrm{~cm}$ from the magnetic grid. The image is magnified using electrostatic lenses to show the fine structure of the grid and is collected using the primary positron beam without time stretching. Besides the spokes of the magnetic grid the
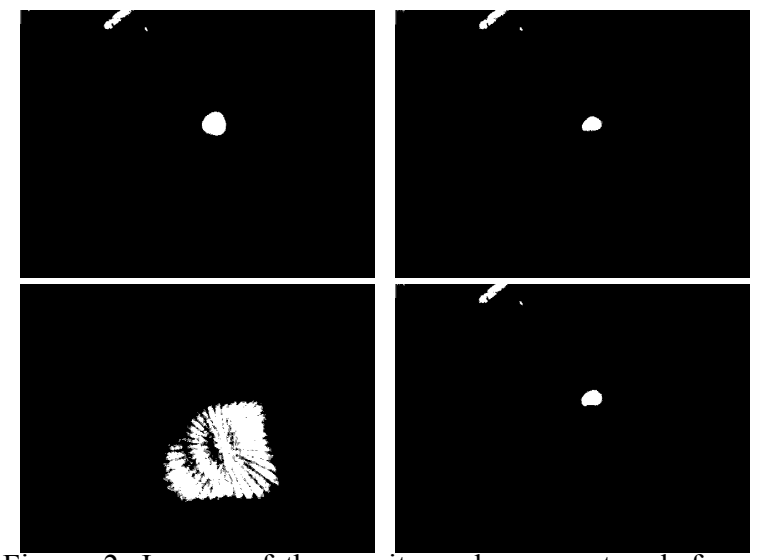

Figure 2. Images of the positron beam captured from a microchannel plate, located $40 \mathrm{~cm}$ from the magnetic grid exit. Images are formed with electrostatic lenses. The images correspond to (starting from to left hand corner, clockwise): Primary beam with the trap turned off, Timestretched positron beam without bunching, Time-stretched and bunched positron beam, Image of the magnetic grid showing the spokes and vane structure. The channel plate active area is $40 \mathrm{~mm}$.

image also shows the vanes of the starting moderator. Since we are able to image the primary positron moderator structure through the magnetic grid, it is clear that the scattering of positrons from the magnetic grid is negligible.

Figure 2 also contains images of focused beam spot corresponding to the primary beam, time-stretched beam, and bunched beam. In all three cases the beam spots remained nearly identical showing the excellent transport properties of the beam through the trap, 
buncher, and magnetic grid systems.

\section{BEAM TRANSPORT}

The stretched beam enables the operation of the positron microprobe and a series of positron spectrographic measurements. A switchyard located 40 $\mathrm{cm}$ from the magnetic grid can divert the electrostatically transported beam to various experiments. The switchyard accommodates three sets of components: A straight through tube that transports positrons to the positron microprobe, a set of cylindrical plates that deflect the positron beam to a multiuser beam feed line, and a microchannel plate for instantaneous beam characterization and tuning.

The user beam lines are designed with electrostatic elements and the beam is diverted to different end stations using more switchyard stations. Experiments actively pursued include high resolution measurements of electron momentum by angular correlation techniques, positron diffraction and holography, positron induced desorption from the surface, and positron induced Auger electron spectroscopy. These techniques have been developed and tested at other facilities and will be attached to the various end stations.

\section{POSITRON MICROPROBE}

LLNL is building a pulsed positron microprobe as a central part of the high intensity positron program. At completion, the microprobe will provide a $100 \mathrm{ps}$ wide bunches of positrons at an intensity of $10^{7}$ positrons/s. The final spot size will be about $1: \mathrm{m}$ and will have a repetition rate of $20 \mathrm{MHz}$. This will allow us to perform positron lifetime spectroscopy to determine the defect size and concentration over spatial volumes of $0.025: \mathrm{m}^{3}$. The energy of the positron beam can be varied $1-50 \mathrm{keV}$ to sample the near surface regions (1-10:m depending on the density of the target material) and buried interfaces.

The positron beam exiting the magnetic grid has the following properties: diameter $=1.2 \mathrm{~cm}$, halfangle of divergence $<1^{\circ}$, energy $=2 \mathrm{keV}$, a pulse width $=1 \mathrm{~ns}$, and a repetition rate $=20 \mathrm{MHz}$. Conversion of this beam into a microprobe is challenging and is achieved using several principles.

The most important principle is the brightness enhancement of the beam using successive stages of moderation. The brightness of a focused positron beam is limited by the phase space constraints set by the primary source. Due to the limitations in the primary positron flux (unlike in an electron microscope, where there is an abundant supply of electrons), collimation is not practical for attaining a

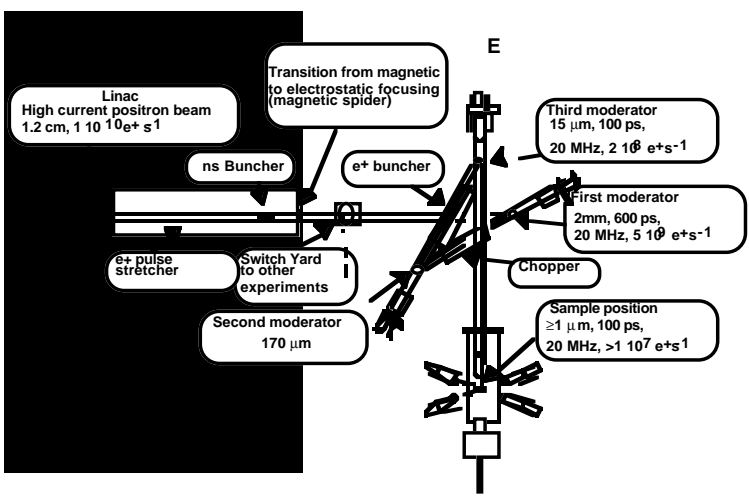

Figure 3. Schematic of the main functional elements of the LLNL 3D-pulsed positron microprobe. The final beam will be $\sim 1: \mathrm{m}$ in diameter and pulsed at $20 \mathrm{MHz}$ with a pulse width of $<100 \mathrm{ps}$. The energy of the beam can be

micron beam spot. The brightness of a positron beam can be increased by several orders of magnitude by taking advantage of the nonconservative forces that are present when a beam of fast positrons $(\sim 5 \mathrm{keV})$ is slowed down in a target and reemitted as slow positrons. ${ }^{3}$ Although, $80 \%$ of the beam is lost in the remoderation process, the brightness enhancement of a remoderation stage is orders of magnitude better than that one can achieve using apertures.

In our design, the final spot size of $\sim 1: \mathrm{m}$ is achieved by three stages of brightness enhancement, each stage producing a compression of $\sim 10$ in diameter. Since we have 3 stages of reflection remoderation, the microprobe can be compartmentalized into four straight sections. The angle between each section is chosen to optimize the overall layout of the microprobe (see Figure 3).

To prevent magnetic field causing unwanted beam modifications in an adjacent section, the beam tranport and focusing is performed using electrostatic elements. All electrostatic elements are designed using the ion simulation program SIMION. The first moderator is located $190 \mathrm{~cm}$ from the magnetic grid. The length of the column is chosen to produce a time focus at the moderator, and reduce the background in the data acquisition window (see below).

The second column is $50 \mathrm{~cm}$ long and produces a spot of $0.15 \mathrm{~mm}$ on the second remoderator. This column contains a chopper section, which is a set of parallel plates $(4 \mathrm{mmH} 4 \mathrm{~mm}, 6 \mathrm{~mm}$ separation) designed to let the positron bunch go through while deflecting the unwanted tails (arising from positrons arriving out-of-phase or leaking out of the stretcher) into an absorber. The chopper receives radio frequency 
voltage timed with respect to the bunches.

The third column (50 cm long) houses the main buncher which will compress the time distribution of the beam from $\sim 1 \mathrm{~ns}$ to $100 \mathrm{ps}$ every 50 ns. The time trigger from the main buncher will be used to obtain the arrival time of the positron bunch at the target. The third column produces a beam spot of $15: m$ on the final moderator.

The final column incorporates elements that are necessary to focus the beam to $\mathrm{a} \sim 1: \mathrm{m}$ spot and to vary the beam energy from $1 \mathrm{keV}$ to $50 \mathrm{keV}$. The final column (113 cm long) contains a buncher section that can be used to provide a tighter time distribution for specialized applications. This column also incorporates a 12-pole deflector lens system to steer the beam across the target. The 12-pole lens also can produce a line focus to scan with greater precision along one dimension.

The components and functions described above have been optimized to yield a set of robust design parameters for the microprobe. The influence of mechanical misallignments of the electrode structures, residual ac magnetic fields, variations in the power supply voltages (ac ripple), and radiation background from successive stages of moderation were all optimized.

The lens elements are distributed to produce an odd number of beam cross-overs in each column, and the cross over positions are adjusted to compensate the beam spot shifts from residual ac magnetic fields. Therefore, a small magnetic field will not alter the position of the electrostatic focus in first order. Simulations show that a one milligauss ac magnetic field broadens the beam spot to $2.6 \mathrm{:m}$. The microprobe columns are housed in a double walled :metal enclosure, and the residual ac magnetic field is expected to be much less than 1 milligauss. It is important to note that a dc background field of 1 milligauss will only shift the final focus spot without degrading the spot size.

The optical column layout and their lengths are optimized to reduce the background radiation in the detector system. Since the bunches are spaced $50 \mathrm{~ns}$ apart, there are several bunches in the system at any given instant. The positron annihilations from moderators, the magnetic grid, accelerating grids, and stopper for the chopped beam can all contribute to the background radiation. The length of the optical columns are chosen to produce all these annihilations in a $10 \mathrm{~ns}$ window before the target annihilations. Thus, we will have a clean data collection time window of $40 \mathrm{~ns}$ that is not disturbed by annihilations from other sources. Since the intensity of $511 \mathrm{keV}$ radiation at the remoderators is several orders of magnitude higher than the target signal itself, this has been an important factor in the layout of various elements.

The location of the radio frequency elements are optimized to reduce the coupling effects. When a bunch receives a radio frequency kick in the chopper, other bunches are located inside well-shielded electrostatic tubes.

Lifetime spectra will be collected using an array of $\mathrm{BaF}_{2}$ detectors. The detectors will be operated in coincidence to prevent background events. The positron backscattering from the target can cause background signal, and is a serious problem in all existing positron beam lifetime systems. To effectively eliminate this background, we have designed our detector system to record only events emanating from a small volume around the target. Each annihilation event is validated by a pair of detectors arranged at 180 degrees which limits validated events to the volume between the two detectors. If an event occurs in the line of sight between the two coincidence detectors, it could be counted as a good event. The enclosure around the target is large enough to discriminate events that originate from the wall.

When completed, the microprobe will be a unique instrument capable of scanning three dimensional images of defect distributions in materials.

\section{CONCLUSION}

A program of new measurements using our intense positron beam is under development at LLNL. The high flux of the LLNL beam and the assortment of spectroscopic tools being developed will enable several unique experiments.

\section{ACKNOWLEDGEMENTS}

This work was performed under the auspices of the US Department of Energy by LLNL under contract No. W-7405-ENG-48.

\section{REFERENCES}

1. See for example, Proceedings of the 11th International Conference on Positron Annihilation, Eds, Y.C. Jean, M. Eldrup, D.M. Schrader, and R.N. West, Materials Science Forum, 255-257, Trans Tech Publications, USA (1997).

2. R. Howell, R.A. Alvarez, and M. Stanek, Appl. Phys. Lett., 40, 751 (1982).

3. A.P. Mills, Jr., Appl. Phys., 23, 189 (1980). 